I Nature, Science \& $\&$ Technology

Research Article

\title{
Microwave Absorption Properties of Unripe Plantain Husk, Polycaprolactone and Charcoal Powder Hybrid Nanocomposites Using Numerical Simulation at X-Band Frequency
}

Abubakar Yakubu, , , , A. Zulkifly ${ }^{2}$, , A, Sirajo ${ }^{3}$

${ }^{1}$ Kebbi State University of Science and Technology, Aliero, Nigeria

${ }^{2}$ Universiti Putra Malaysia, Sri-Serdang, Selangor, Malaysia

${ }^{3}$ Kebbi State Universiti of Science and Technology, Aliero, Nigeria

\section{Keywords}

Unripe plantain husk powder, Charcoal,

Dielectric properties, filler,

Shielding effectiveness.

\begin{abstract}
Single bulk materials have lesser advantages compared to nano materials in the area of mechanical, electrical, dielectric, optical and thermal properties enhancement of polymer and hybrid of bio-composites. In this work, charcoal (CH), unripe plantain husk (UPH), and polycaprolactone (PCL) based microwave absorber were synthesized using both mechanical alloy and melt blend techniques, respectively. Different percentages of the prepared charcoal nanoparticles was introduced into the hybrid composites to study the effect on dielectric properties, shielding effectiveness, reflection loss and structural morphology in the frequency range of 8-12 GHz.. Findings showed that the sample with the highest amount of charcoal percentage had the best shielding effectiveness and dielectric constant. The value of dielectric constant gradually increased from 3.06 to 5.45 at the frequency range measured. The charcoal average particle size was estimated to be $21.42 \mathrm{~nm}$ using TEM analysis while the mean deviation of surface roughness ranged from 5.45 to $25.56 \mathrm{~nm}$ using atomic force microscope. The highest shielding effectiveness of the hybrid composites was obtained for the $30 \mathrm{wt} \%$ nano charcoal content with a value of $-48.78 \mathrm{~dB}$ at $10 \mathrm{GHz}$. The complete dispersion and interaction of the charcoal with the PCL and UPH enhanced the dielectric constant, loss factor, hence making it potent in microwave absorption, shielding and radar applications.
\end{abstract}

\section{Introduction}

Most electronics and telecommunication industries use ferrites as microwave absorbers. Ferrites popularity as microwave absorbers is attributed to their unique electrical sensitivity [1]. The number of researches on EM wave's absorbers using ferrites is considerably high. However, ferrites are dangerous to both human and the environment. It is on this note that researches started reacting ferrites with polymer so as to probably reduce its effect on the environment. The current quest is to look for microwave-absorbing material (MAM) and radar absorbing materials (RAM) that have thin thickness, lightweight, wide bandwidth, and are cost effective which can eradicate EM interference $[2,3]$.

The flexibility and complex permittivity of polycaprolactone (PCL) makes it one of the choice polymer in fabrication EM absorber to any desired shape. Most researchers have reported the use of ferrites with fibers of oil palm and other materials [4]. Agricultural residue that are biodegradable, eco-friendly and cost effective can be used to fabricate microwave absorbers [5].

In [6], it was reported that higher carbon content in agricultural waste increases the waste ability to attenuate electromagnetic wave and that microwave absorber can be synthesized via agricultural waste single-layer absorber with low thickness. Shown in Table 1 and 2 are the constituents and physiochemical properties of UPH powder, respectively.

For this study, a single layer hybrid nanocomposites was prepared using three different materials namely charcoal (CH), PCL and unripe plantain husk (UPH). The choice of materials in this work was based on the properties of the materials and the availability of the UPH which is biodegradable and abundantly available.
Table 1: Constituents of UPH husk powder

\begin{tabular}{lc} 
Constituent & Percentage (\%) \\
Hemicellulose & 40.1 \\
a-Cellulose & 37.5 \\
Hollocellulose & 72.3 \\
Water soluble components & 14.0 \\
Lignin & 8.1 \\
\hline
\end{tabular}

Challenges raised on ferrites may possibly be addressed by reinforcing $\mathrm{UPH} / \mathrm{PCL}$ composites with $\mathrm{CH}$. The addition $\mathrm{CH}$ to the UPH/PCL composites will increase the hybrid interfacial adhesion and interaction between materials. The method of combining more than two materials to form composites is the new area of materials engineering and this is achieved through different synthesis route. The proposed hybrid composites can be used in microwave devices, shielding effectiveness, microelectronics and in communication industries. For better understanding of the proposed material, exploiting its electromagnetic properties is vital.

Table 2: Physicochemical Properties of UPH Powder

\begin{tabular}{lc}
\hline Constituent & Percentage (\%) \\
Volatile matter & 2.05 \\
Moisture content & 12.67 \\
Ash content & 8.10 \\
Carbon content & 67.65 \\
\hline
\end{tabular}

The main focus in our work is to change ferrite that is hazardous to nature and produce a good microwave absorber which would be cheaper, lighter and improve permittivity, flexibility and absorptivity 
of the hybrid composites produced and to examine impact of various charcoal loadings on properties of the hybrid nanocomposite which was hitherto absent in earlier composites. COMSOL Multiphysics, finite element method (FEM) will be used to numerically calculate shielding effectiveness and visualize the electric field distribution of the composites using the geometry of a microstrip antenna.

\section{Background theory}

Attenuation $(\mathrm{dB})$ is defined as a ratio between impinging energy to residual energy, when EM passes through a shield medium both reflection and absorption are obtained. The incident and transmitted wave are mathematically represented as $|\mathrm{S} 11|$ and $|\mathrm{S} 21|$, respectively. For microstrip measurement, power-loss in air and in sample is given as [7];

$$
\begin{aligned}
& P(\text { loss air })=1-\left(S_{11}\right)^{2}-\left(S_{21}\right)^{2} \\
& P(\text { loss sample })=1-\left(S_{11}\right)^{2}-\left(S_{21}\right)^{2}-P(\text { loss air }) \\
& \text { Absorption }=P(\text { loss sample })-P(\text { loss air })
\end{aligned}
$$

Attenuation of the radiation propagation through the material can be calculated using;

$$
\text { Attenuation }(d B)=20 \log \left(S_{21}\right)
$$

When a plane wave is incident on a shielding material, the total shielding effectiveness (SE) is computed from the reflected wave and the transmitted wave, hence SE is given as [8];

$$
\begin{aligned}
& S E=S E_{A}+S E_{R} \\
& S E_{A}=-10 \log \left(\left(S_{21}\right)^{2} / 1-\left(S_{11}\right)^{1}\right) \\
& S E_{R}=-10 \log \left(1-S_{11}\right)
\end{aligned}
$$

The reflection or return loss can be expressed in terms of reflected power and incident power as $R L(d B)=$ $20 \log \left(P_{r} / P_{i}\right)$

The complex relative permittivity is given as,

$$
\varepsilon=\varepsilon^{\prime}-j \varepsilon^{\prime \prime}
$$

where $\varepsilon^{\prime}$ is the dielectric constant $\varepsilon^{\prime \prime}$ and is the loss factor.

The transmission and reflection coefficients of a two-port network are defined in terms of the wave variables as [9, 10];

$$
\begin{array}{ll}
\left.S_{11}=\frac{b_{1}}{a_{1}}\right]_{a_{2}=0} & \left.S_{12}=\frac{b_{1}}{a_{2}}\right]_{a_{1}=0} \\
\left.S_{21}=\frac{b_{2}}{a_{1}}\right]_{a_{2}=0} & \left.S_{22}=\frac{b_{2}}{a_{2}}\right]_{a_{1}=0}
\end{array}
$$

Where, $a_{n}=0$ implies a perfect impedance match (no reflection from terminal impedance) at port $n$. The parameters $S_{11}$ and $S_{22}$ are called the reflection coefficients, whereas $S_{12}$ and $S_{21}$ are called the transmission coefficients. These are the parameters directly measurable at microwave frequencies.

\section{Materials and Methods}

\subsection{Charcoal preparation}

Charcoal powder used in this study was obtained commercially at Sigma Aldrich with purity of $99.9 \%$. The charcoal powder was then milled in a high energy mill using SPEX 8000D shaker for 8 hours. The ball to powder mass ratio used was $5: 1$. The milled powder was then set for further experiment.

\subsection{UPH powder preparation/PCL}

Commercial grade polycaprolactone (PCL) was supplied by Sigma Aldrich. The melt flow index, density and melting temperature are $12.5 \mathrm{~g} / 10 \mathrm{~min}, 1.145 \mathrm{~g} / \mathrm{cm}^{3}$, and $70{ }^{\circ} \mathrm{C}$, respectively. UPH powder was prepared by washing the husk (UPH) with water and dipping the washed husks into acetone for a period of 5 minutes before Sun drying. The husk was sundried for 48 hours before moving it to a local grinding machine. The grinded UPH powder is then sieved to a size of $200 \mu \mathrm{m}$.

\section{3. $\mathrm{CH} / \mathrm{PCL} / \mathrm{UPH}$ hybrid nanocomposites Preparation}

Nano $\mathrm{CH}, \mathrm{PCL}$ and UPH were weighed according to the required proportion as highlighted in Table 3.

\section{Table 3: Compositions of Materials Used}

\begin{tabular}{lllllll}
\hline \multicolumn{2}{c}{ Nano CH } & UPH & \multicolumn{3}{c}{ PCL } & $\begin{array}{l}\text { Total } \\
\text { (g) }\end{array}$ \\
\cline { 1 - 5 }$(\%)$ & (g) & $(\%)$ & $(\mathrm{g})$ & $\mathbf{( \% )}$ & $\mathbf{( g )}$ & \\
$\mathbf{5 . 0}$ & 1.0 & 40 & 8.0 & 55 & 11.0 & 20 \\
$\mathbf{1 5 . 0}$ & 3.0 & 50 & 10.0 & 35 & 7.0 & 20 \\
$\mathbf{2 5 . 0}$ & 5.0 & 60 & 12.0 & 15 & 3.0 & 20 \\
$\mathbf{3 0 . 0}$ & 6.0 & 65 & 13.0 & 5 & 1.0 & 20 \\
\hline
\end{tabular}

The composite of nano CH-PCL-UPH was prepared via the Thermo Haake blending machine. The machine was set to $90{ }^{\circ} \mathrm{C}$, the rotor speed of the blend was set at $50 \mathrm{rpm}$. After the vial of the blend has reached $90{ }^{\circ} \mathrm{C}$, proportion of PCL is first placed in vial and allowed to melt before adding proportions of nano $\mathrm{CH}$ and UPH powder into the vial. The mixture is allowed to blend for $15 \mathrm{mins}$ before being removed from the vial and then fabricated into pellets. The pellets were prepared by placing $20 \mathrm{~g}$ of the blends into a mold with the dimensions 10 by $8 \mathrm{~cm}^{2}$, with thickness of $8 \mathrm{~mm}$ and preheated for 10 mins between plates at $80^{\circ} \mathrm{C}$. Voids and bubbles in the samples were avoided by allowing a breathing time of 10 seconds. The sample was then pressed at a pressure of $110 \mathrm{~kg} / \mathrm{cm}^{2}$ for $10 \mathrm{mins}$. Finally rectangular pellets of $8 \mathrm{~mm}$ thickness were then fabricated after cooling. The produced substrates were then used for characterization.

\section{Experimental \\ 4.1. Complex permittivity measurement}

In this technique, measurements of the dielectric constant and the loss factor were carried out using coaxial probe connected via a coaxial cable, to an Agilent vector network analyzer N5230A PNA-L. A one-port reflection-only calibration was performed using a 3.5 inch shorting block and distilled water at room temperature. The calibration was verified by measuring the permittivity values for water which is standard material. The result obtained for water is compared to standard value. Good agreement between the results affirms accuracy of calibration. The measurement technique using vector network analyzer is shown in Fig. 1.

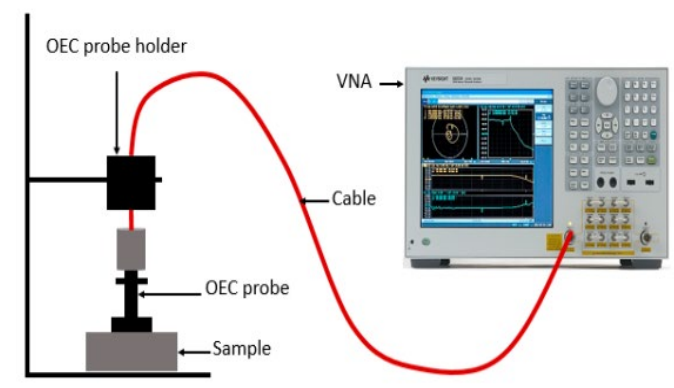

Figure 1. Complex Permittivity Measurement Set-up [11]

4.2 Scattering Parameter Measurement 
Finite element method (FEM) was used to simulate a microstrip antenna which was used to numerically calculate reflection loss, shielding effectives and visualize electric field distribution. A single $\mathrm{TE}_{10}$ mode wave propagating through the microstrip was chosen for the simulation. The complex permittivity of the RT duroid 5880 used in simulation is $2.2-j^{\star} 0.0008$ while the substrate dimension is $10.0 \mathrm{~cm}$ long, $8.0 \mathrm{~cm}$ wide and $8 \mathrm{~mm}$ thick and with a signal line (width $=1.5$ $\mathrm{mm}$, length $=8.0 \mathrm{~cm}$ ) etched on the surface of the microstrip.

\section{Results}

\subsection{FTIR analysis}

The peaks for the CH-PCL-UPH hybrid nano composites, PCL and nano $\mathrm{CH}$ is shown in Fig. 2. Observation shows that the nano $\mathrm{CH}$ have the highest absorption intensity while PCL had the lowest absorption intensity. However, the hybrid composites gave rise to spectral of different intensities. At the selected wavelength range, the $730 \mathrm{~cm}^{-1}$ peak is due to long chain rocking vibrations, the $1200 \mathrm{~cm}^{-1}$ peak due to C-O-C aliphatic ether stretching vibrations [12].

The principal bands of pure PCL is 1467 and $1362 \mathrm{~cm}^{-1}\left(\mathrm{CH}_{2}\right.$ and $\mathrm{CH}$ bending vibrations) $1292-1042 \mathrm{~cm}^{-1}$ (C-O-C aliphatic ether stretching vibrations), and $730 \mathrm{~cm}^{-1}$ ( $\mathrm{CH}_{2}$ long chain rocking motion vibrations) [13]. The spectral simply confirmed that the addition of the nanoparticles of charcoal into the hybrid significantly affected the absorption pattern of the hybrid composites. The charge transfer interactions between the conjugated surfaces of the charcoal and the particles of PCL/UPH significantly modified the properties of the composites [14].

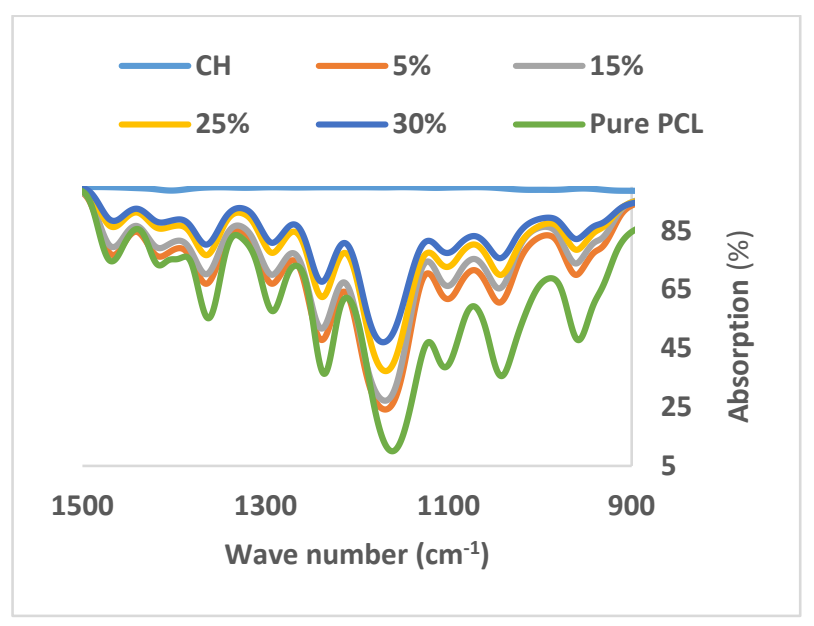

Figure 2. FTIR Spectral for Different (\%) of Filler

\subsection{Transmission electron microscope}

Fig. 3 depicts the surface morphology of nano $\mathrm{CH}, \mathrm{PCL}$ and $\mathrm{CH} / \mathrm{PCL} / \mathrm{UPH}$ hybrid nano composites. Evidence of uniform shapes are shown in the micrographs of nano $\mathrm{CH}$. The average particle size of the nano $\mathrm{CH}$ is estimated to be $21.42 \mathrm{~nm}$ using TEM and Image $\mathrm{J}$ software. The micrograph of the CH-PCL-UPH hybrid nano composites shows strong evidence of nanoparticles dispersion in the composites. In addition, there was strong interfacial interaction between the UPH powder and PCL which in turn strengthen the adhesive bond between $\mathrm{UPH} / \mathrm{PCL}$ and nano $\mathrm{CH}$. The uniform dispersion of the filler particles in the hybrid composites created a bond which may strengthen composite electromagnetic and conductive properties [15]. The large surface area of the hybrid nano-composites are due to the nano particles cohesion and compactness in the matrix which is expected to contribute to the material interfacial polarization leading to reduction of EM waves $[16,17]$.

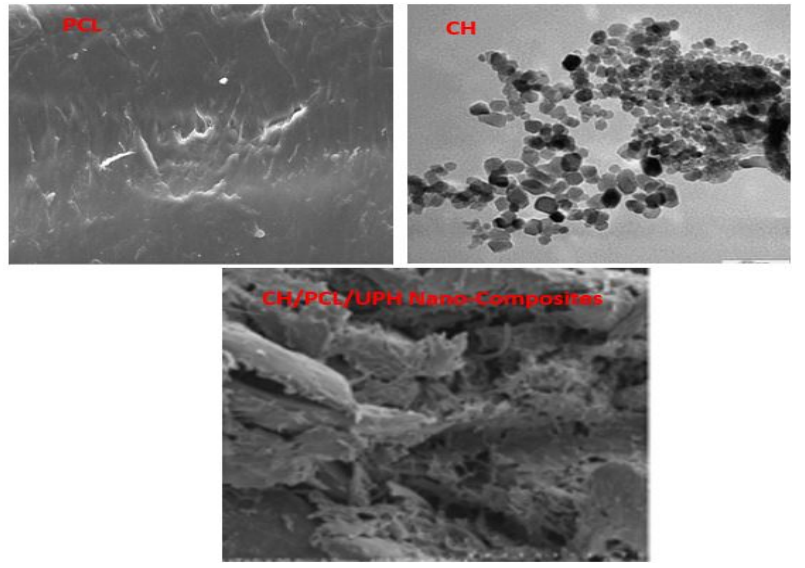

Figure 3. Surface Morphology of all Samples

\subsection{Dielectric property}

The graphs shown in Figs. 4 and 5 represents the dielectric constant and loss factor of the composites, respectively. Careful observation on the graphs showed that both the dielectric constant and loss factor increased as filler content increases in the hybrid composites. The increase in the dielectric constant is expected based on the effective medium theory, where dielectric enhancement of polymer materials can be achieved by doping with materials with higher dielectric constant than the polymer [18]. Further observation showed that the addition of $30 \%$ nano loadings into the hybrid composites produced the highest values for both the dielectric constant and loss factor. At $10 \mathrm{GHz}$, the dielectric constant values for PCL, 5, 15, 25 and $30 \%$ fillers and $\mathrm{CH}$ nanoparticles, were $3.06,3.46,3.61,3.75,4.54$ and 5.45, respectively. Table 4 illustrates the change in values of dielectric constant and loss factor with addition of the nano loadings.

Table 4: Average Relative Permittivity of CZF/PCL/UPH Nano Composites

\begin{tabular}{ll}
\hline Sample (\%) & Relative Permittivity \\
\hline PCL & $3.06-j^{\star} 0.29$ \\
5.0 & $3.46-j^{\star} 0.43$ \\
15.0 & $3.61-j^{\star} 0.50$ \\
25.0 & $3.75-j^{\star} 0.60$ \\
30.0 & $4.54-j^{\star} 0.76$ \\
CH & $5.45-j^{\star} 0.99$ \\
\hline
\end{tabular}

Since the material produced is heterogeneous in structure, the change in loss factor is associated to changing field polarization. As frequency increases on the scale, there is a systematic change in electric field causing non ion diffusion in the field direction resulting in decrease in loss factor as frequency increases [19]. 


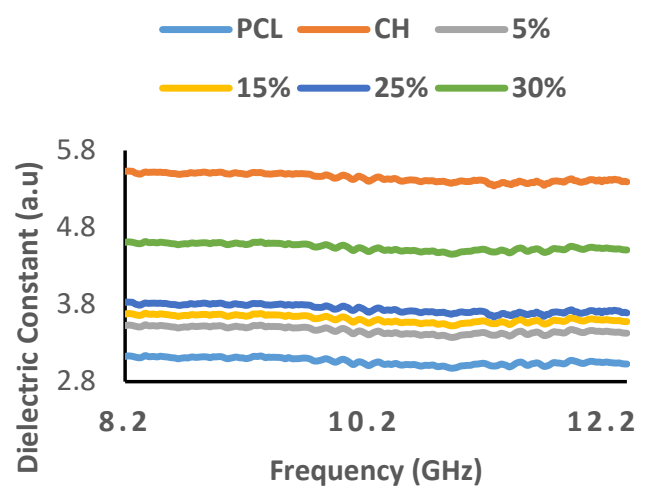

Figure 4. Dielectric constant for $\mathrm{CH} / \mathrm{PCL} / \mathrm{UPH}$ hybrid nano composites

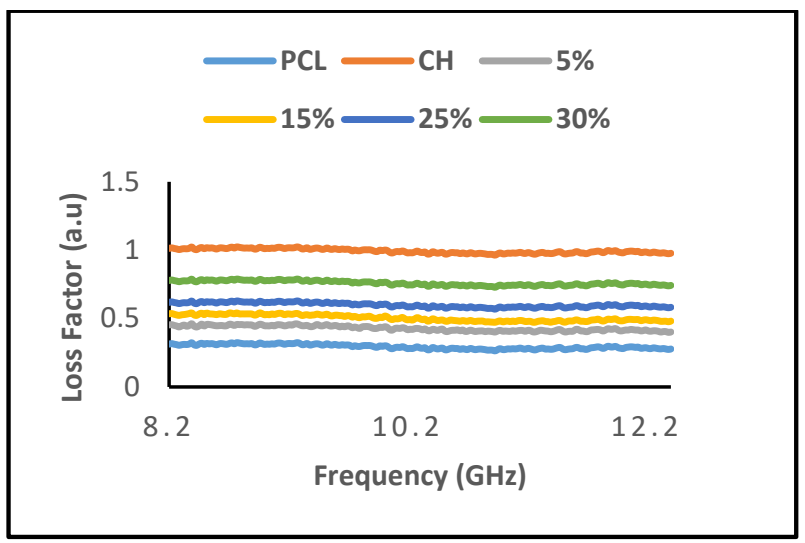

Figure 5: Loss Factor for CH/PCL/UPH hybrid nano composites

\subsection{Shielding effectiveness (dB)}

Observation on Fig. 6 showed that the hybrid composites had an appreciable amount of shielding effectiveness total $\left(\mathrm{SE}_{\mathrm{T}}\right)$ as filler composition increases in the hybrid. The lowest value of shielding effectives at $10 \mathrm{GHz}$ was recorded for the lowest filler while the highest was recorded for the highest filler inclusion. There was a sequential increase in the SE from -10.40 to $-48.78 \mathrm{~dB}$ at $10 \mathrm{GHz}$. The highest SE attributed to the $30 \% \mathrm{CH}$ nano filler was due to its specific high surface area and interfacial adhesion and cohesion with the matrix [20].

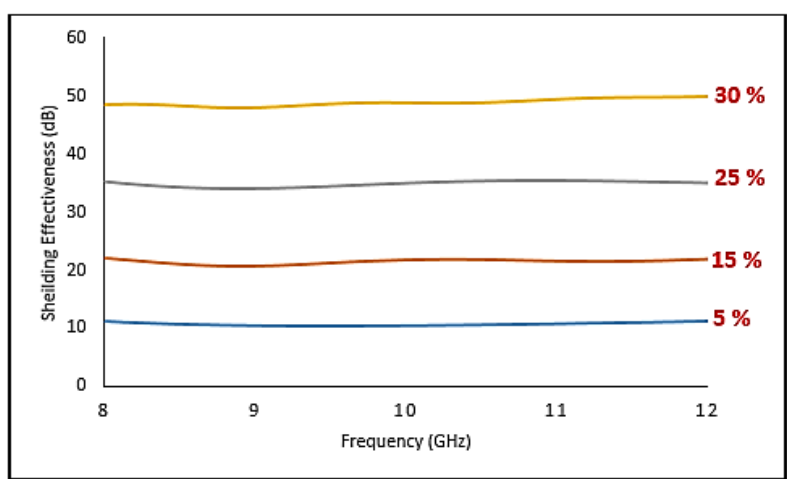

Figure 6: SE for CH/PCL/UPH hybrid nano composites

\subsection{Electric field distribution}

Fig. 7 revealed that material permittivity plays a determinant role in the pattern of the field obtained for each filler percent. The green color represents field intensity propagating from the input to output port of the microstrip antenna. The higher the dielectric constant, the less energy at the output due to reflection at the input and absorption at the output. It is observed that as the filler loading increases higher absorption of EM waves was obtained. It is expected that higher loss materials tend to absorb more of EM waves [21]. The result is in agreement with the result of the SE obtained for the different fillers percentages.

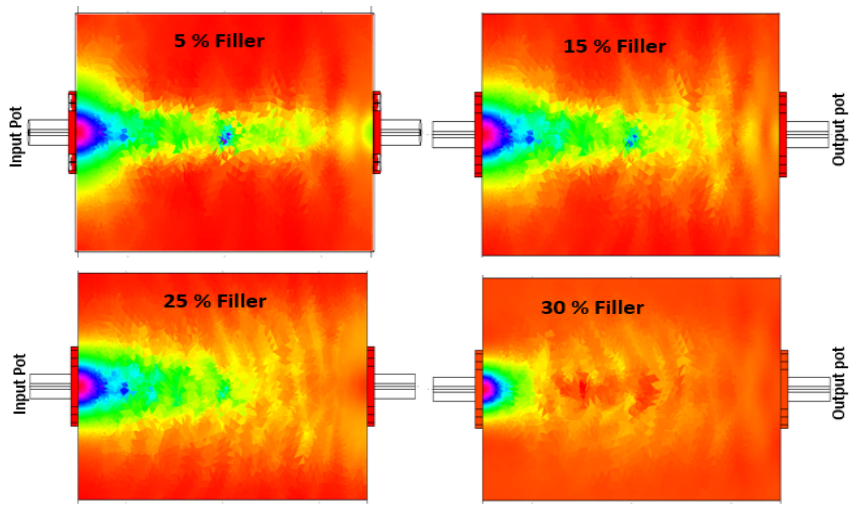

Figure 7. Electric Field Distribution for Different (\%) of CH/PCL/UPH nanocomposites

\subsection{Reflection loss}

The numerically calculated refection loss for all the samples is shown in Fig. 8. It can be seen that all samples demonstrated significant absorption between 9 to $10 \mathrm{GHz}$, The $30 \%$ charcoal filler had the highest return loss of $-33.5 \mathrm{~dB}$ at the point considered. The absorption value for the $30 \%$ filler is very significant in which more energy was absorbed. This behavior is attributed to impedance matching between the absorber surface and free space, thus minimizing refection at the front-face [22]. The impedance matching ensures that the EM waves enter the absorber optimally at the surface avoiding surface parallax of EM propagation. Further observation showed that a sinusoidal loop is noticed which excites both the electric and a magnetic field, respectively leading to a strong localized EM field at the resonance frequency. The result obtained for the return loss is in complete agreement with the result obtained for the SE and electric field distribution shown in Figs. 6 and 7, respectively.

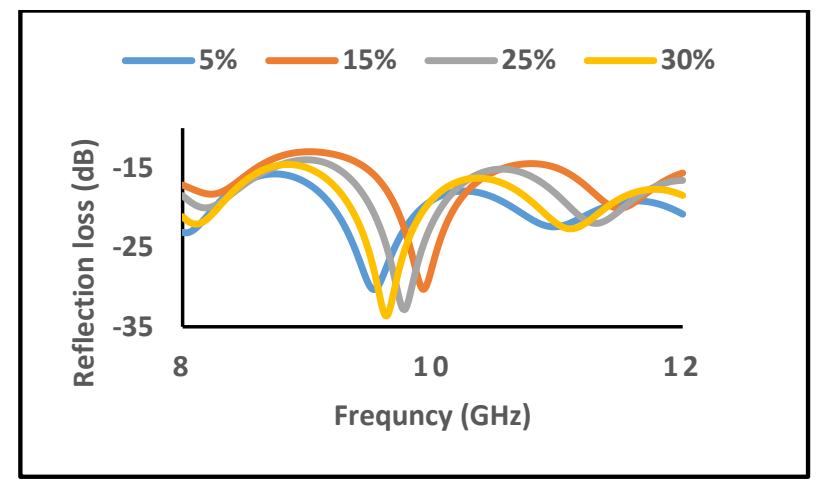

Figure 8: Reflection loss of composite

5.7 Atomic force microscope

The surface of the fabricated rectangular pellets were measured at a horizontal scale of $2 \mu \mathrm{m}$ and vertical scale of $50 \mathrm{~nm}$. Careful observation on Fig. 9 depicts infinitesimal grooves and boundaries in all filler percent. Based on the measurement analysis, the lowest filler (\%) has a mean deviation of $22.3 \mathrm{~nm}$ surface smoothness while the filler with the highest filler (\%) has a mean deviation $5.65 \mathrm{~nm}$. The difference in the surface roughness as filler increases is attributed to increase surface area resulting in a compact dense surface [23]. Based on the AFM analysis, the surface roughness of the samples is considered smooth since the roughness was in $\mathrm{nm}$ scale proposing that the samples are good for electromagnetic wave characterization. 

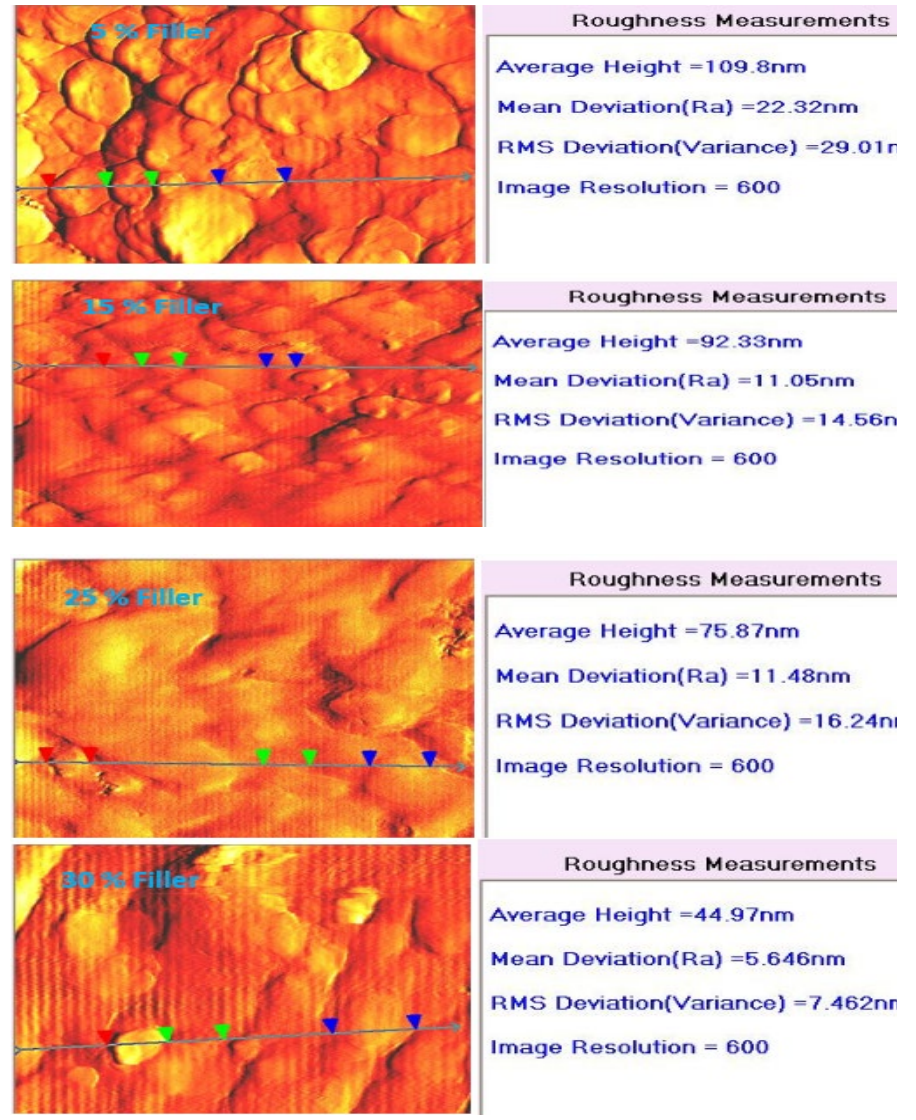

Roughness Measurements

Average Height $=75.87 \mathrm{~nm}$

Mean Deviation $(\mathrm{Ra})=11.48 \mathrm{~nm}$

RMS Deviation(Variance) $=16.24 \mathrm{n}$

Image Resolution $=600$

Roughness Measurements

Average Height $=44.97 \mathrm{~nm}$

Mean Deviation $(\mathrm{Ra})=5.646 \mathrm{~nm}$

RMS Deviation(Variance) $=7.462 \mathrm{n}$

Image Resolution $=600$

\section{Figure 9: Surface Roughness Composites}

6. Conclusion

The prepared charcoal nano via high energy ball milling was mixed with PCL and UPH powder via blending using the Thermo Haake extruder. Using TEM, SEM and FTIR, the CH-PCL-UPH hybrid composites was structurally investigated and result showed homogenous dispersed filler in matrix, where the average particle size obtained $21.72 \mathrm{~nm}$, while the $30 \%$ filler particles produced a shielding of $-48.3 \mathrm{~dB}$ and a highest complex permittivity of $4.54-\mathrm{j}^{\star} 076$. Based on the value of the dielectric loss obtained at the measured frequency, the material has potential to absorb electromagnetic wave at microwave frequency. There was enhancement in the dielectric constant of the composites when filled with nano particles of $\mathrm{CH}$. The nano filler also improve the shielding effectiveness of the hybrid composites, making it suitable for small aircraft radiation cover shield.

\section{Declaration of Conflict of Interests}

The author(s) declare(s) that there is no conflict of interest. They have no known competing financial interests or personal relationships that could have appeared to influence the work reported in this paper.

\section{Acknowledgement}

The authors wish to thank the Department of Physics, Kebbi State University of Science and Technology, Aliero, Nigeria and the Department of Physics,

Universiti Putra Malaysia for providing facilities for the research.

\section{References}

[1.] Mensah, E.E., Abbas, Z., Azis, R.A.S. and Khamis, A.M Enhancement of Complex Permittivity and Attenuation Properties of Recycled Hematite ( $\alpha$-Fe2O3) Using Nanoparticles Prepared via Ball Milling Technique. Materials,12, (2019), 1696. https://doi.org/10.3390/ma12101696
[2.] Panwar, R., \& Lee, J. R. Recent advances in thin and broadband layered microwave absorbing and shielding structures for commercial and defense applications. Functional Composites and Structures, 1(3), (2019), 032001.

[3.] Setua, D. K., Mordina, B., Srivastava, A. K., Roy, D., \& Prasad, N. E. Carbon nanofibers-reinforced polymer nanocomposites as efficient microwave absorber. In Fiber-Reinforced Nanocomposites: Fundamentals and Applications, (2020), (pp. 395-430). https://doi/.org/10.1016/B978-0-12-819904-6.00018-9

[4.] Fahad A. Abbas, Z. Obaiys. S. Yakubu, A. Dielectric Behavior of OPEFB Reinforced Polycaprolactone Composites at X-Band Frequency. International Polymer Processing Journal of the Polymer Processing Society. 31(1), (2016), :18-25, https://doi:10.3139/217.3069

[5.] Yakubu, A Abbas, $Z$ and Abdullahi, S. Mechanical, Dielectric and Shielding Performance of Rice Husk/Polycaprolactone Composites Enhanced via Rice Husk Particles Inclusion, Open Access Library Journal, 7(7), (2020a),1-12. https://doi.org/10.4236/oalib.1106514

[6.] Pattanayak, S. S., Laskar, S. H., \& Sahoo, S. Microwave absorption performance enhancement of corn husk-based microwave absorber. Journal of Materials Science: Materials in Electronics (2020a), 1-11. https://doi.org/10.1007/s10854-020-04888-1

[7.] Yakubu, A., Abbas, Z., \& Sirajo, A. Attenuation and Shielding Performance of Wood-Polymer Composites Synthesized via MeltBlend Technique. Open Access Library Journal, 7(2), (2020b) 1-11. https://doi.org/10.4236/oalib.1105444

[8.] Gahlout, P. and Choudhary, V. EMI shielding response of polypyrrole MWCNT/polyurethane composites. Synthetic Metals. (2020), 05-19, https://doi:10.1016/j.synthmet.2020.116414

[9.] Abbas, Z. Pollard, R. D. and Kelsall, R. W. Complex Permittivity Measurements at $\mathrm{Ka}$ Band Using Rectangular Dielectric Waveguide. IEEE Transaction on Instrumentation and Measurement, Vol. 50, No. 5, (2001), pp 1334-1342

[10.] Essaadali, R., Jarndal, A., Kouki, A. B., \& Ghannouchi, F. M. On the Accurate Voltage and Current Analytical Relationship to SParameters of a Nonlinear Two-Port Network. IEEE Transactions on Microwave Theory and Techniques, 66(10), (2018), 4439-4451.

[11.] Ismail I. L, Zulkifly A, Syahidah Azis, R, Ibrahim, N. A and Abd Rahman, M. A. The Effect of MWCNTs Filler on the Absorbing Properties of OPEFB/PLA Composites Using Microstrip Line at Microwave Frequency, Materials, 13, (2020), 4581, https://doi:10.3390/ma13204581

[12.] Viljoen, W. D., \& Labuschagne, F. J. W. J. The thermal stability of highly filled high-density polyethylene quaternary composites: Interactive effects and improved measures. Polymer Testing, 85, (2020), https://doi.org/10.1016/j.polymertesting.2020.106424

[13.] Gureva, S. A., \& Marikhin, V. A. Investigation of structural transformations of long-chain molecular crystals during phase transitions by IR-spectroscopy. In Journal of Physics: Conference Series Vol. 1236 No. 1, (2019),p. 012008). https://doi/10.1088/1742$6596 / 1236 / 1 / 012008$

[14.] Pattanayak S. S, Laskar S. H, Sahoo S. Microwave absorption study of dried banana leaves-based single-layer microwave absorber. International Journal of Microwave and Wireless Technologies. (2020b), https://doi.org/10.1017/S1759078720000707

[15.] Tessema, A., Zhao, D., Moll, J., Xu, S., Yang, R., Li, C., ... \& Kidane, A. Effect of filler loading, geometry, dispersion and temperature on thermal conductivity of polymer nanocomposites. Polymer Testing, 57 , (2017),101-106 https://doi/10.1016/j.polymertesting.2016.11.015

[16.] Ravindren, R.; Mondal, S.; Nath, K.; Das, N.C. Synergistic effect of double percolated co-supportive MWCNT-CB conductive network for high-performance EMI shielding application. Polymer Advanced Technologies, 30, (2018) 1506-1517. https://doi.org/10.1002/pat.4582

[17.] Zhang, Y., Si, H., Liu, S., Jiang, Z., Zhang, J., \& Gong, C. Facile synthesis of BN/Ni nanocomposites for effective regulation of microwave absorption performance. Journal of Alloys and Compounds,850, (2021), 156680. https://doi.org/10.1016/j.jallcom.2020.156680

[18.] Bikky, R., Badi, N., \& Bensaoula, A. Effective medium theory of nano-dielectrics for embedded energy storage capacitors. In COMSOL Conference. (2010)

[19.] Jiang, D., Murugadoss, V., Wang, Y., Lin, J., Ding, T., Wang, Z., ... \& Guo, Z. Electromagnetic interference shielding polymers and 
nanocomposites-a review. Polymer Reviews, 59(2), (2019), 280337. https://doi.org/10.1080/15583724.2018.1546737

[20.] Tran, L. Q. N., Yuan, X. W., Bhattacharyya, D., Fuentes, C., Van Vuure, A. W., \& Verpoest, I. Fiber-matrix interfacial adhesion in natural fiber composites. International Journal of Modern
Physics
(2015),
29(10n11)
1540018.

https://doi.org/10.1142/S0217979215400184

[21.] Pozar D. M. Microwave Engineering. John Wiley \& Sons; U.S.A. (2012)

[22.] Ibrahim, I. R., Matori, K. A., Ismail, I., Awang, Z., Rusly, S. N. A., Nazlan, R., ... \& Ertugrul, M. A Study on Microwave Absorption Properties of Carbon Black and Ni0.6Zn0.4Fe2O4 Nanocomposites by Tuning the Matching-Absorbing Layer Structures. Scientific reports, 10(1), (2020), 1-14. https://doi.org/10.1038/s41598-020-60107-1

[23.] Yakubu, A., Abbas, Z., Olugbenga, O., \& Sahabi, S. Influence of Nano Particle Size on Attenuation and Dielectric Properties of Plantain Husk Powder Using Microwave Techniques at X-Band Frequency. Physical Science International Journal, 24(5), (2020c), 41-51. https://doi.org/10.9734/psij/2020/v24i530192

\section{How to Cite This Article}

Yakubu, A., Zulkifly, A., and Sirajo, A., Microwave Absorption Properties of Unripe Plantain Husk, Polycaprolactone and Charcoal Powder Hybrid Nanocomposites Using Numerical Simulation at X-Band Frequency, Journal of Nature, Science \& Technology, 2(2021), 1-6.

https://doi.org/10.36937/janset.2021.002.001 